\title{
Performance Comparison of AOdv AND OlsR USING 802.11A AND DSRC (802.11P) PROTOCOLS IN URBAN VANETS
}

\author{
Ederval Pablo Ferreira da Cruz ${ }^{1}$ and Bruno dos Santos Silva ${ }^{2}$ \\ ${ }^{1}$ Federal Institute of Espirito Santo (IFES) - Campus Itapina, Colatina-ES, Brazil \\ ${ }^{2}$ Federal University of State of Rio de Janeiro (UNIRIO), Rio de Janeiro-RJ, Brazil
}

\begin{abstract}
A Vehicular Ad Hoc Network (VANET) is a network formed purely among vehicles without presence of any communication infrastructure as base stations and/or access point. Frequent topological changes due to high mobility is one of the main issues in VANETs. In this paper we evaluate Ad-hoc On-Demand Distance Vector (AODV) and Optimized Link State Routing (OLSR) routing protocols using 802.11 a and 802.11p in a realistic urban scenario. For this comparison, we chose five performance metrics: Path Availability, Endto-End Delay, Number of Created Paths, Path Length and Path Duration. Simulation results show, that for most of the metrics evaluated, OLSR outperforms AODV when 802.11p and that 802.11p is more efficient in urban VANETs.
\end{abstract}

\section{KEYWORDS}

OLSR, AODV, Vehicular Networks, VANET, Performance \& $802.11 \mathrm{p}$

\section{INTRODUCTION}

In 1999, the Federal Communication Commission (FCC) defined to allocate a frequency spectrum for vehicle-to-vehicle (V2V) and vehicle-to-roadside (V2R) wireless communication. A public safety and private applications communication service that uses the 5.850-5.925 GHz band (or simply $5.9 \mathrm{GHz}$ band) was established by the FCC in 2003. Such service is called Dedicated Short Range Communications (DSRC) or 802.11p [1,2] where the vehicles and beacons on roadsides can form a Vehicular Ad Hoc Network (VANET). Several research groups and automotive makers consider VANET an important technology for improving passenger's safety avoiding more accidents and deaths, efficiency of the actual transportation system and great opportunities in commercial applications $[3,4,5]$.

VANET consists of vehicles with radio-enabled equipments acting as mobile nodes and/or routers for others nodes, with no existing pre-established infrastructure where such nodes connect themselves in a decentralized, self-organizing manner and also establish multi hop routes. In a VANET, it is possible to exchange information among vehicles, allowing the development of new applications, introducing new services such as on-board-entertainment during the travels. Furthermore, this technology can improve passengers' safety by implementing active safety systems (e.g. emergency breaking according to the traffic received) or automatic emergency calls when an accident occurs.

VANET has some unique characteristics that distinguish it from other kinds of mobile ad hoc networks. 
- Very dynamic topology: Vehicles move in high speed and with different mobility patterns, reflecting in constant changes on the topology of VANETs.

- Partitioned network and reduced contact time: Due to dynamic topology, the connectivity of the VANETs can change frequently, creating a partitioned network, interfering the communication of the nodes, especially when the vehicles density is low.

- High range transmission: The DSRC service allows a range transmission up to 1000 meters.

Therefore, VANETs have some characteristics that make it very challenging. One of these challenges is routing data between sender-destination nodes in a multi hop way.

Basically, the proposed protocols to VANET are classified in two groups [6]: 1) topology-based protocols and 2) geography-based protocols. In this paper we compare two classics mobile networks routing protocols: Optimized Link State Routing (OLSR) and Ad Hoc On-Demand Distance Vector (AODV) using the 802.11a and 802.11p. The main contributions of our work when compared to the existing literature are the following: 1) We measure the performance of $802.11 \mathrm{p}$ and 802.11a in a realistic urban scenario. 2) We also evaluate other performance metrics when compared with other papers.

\section{RELATED WORKS}

Authors in [7] compare and evaluate performance of Ad-hoc On-Demand Distance Vector (AODV), Dynamic Source Routing (DSR) and Swarm Intelligence based protocol. A variety of simulations for VANET is performed, characterized by networks' mobility and size.

In [8] transmission probabilities of $802.11 \mathrm{p}$ are modelled evaluating the effects of these probabilities in DSR, Fish-Eye State Routing (FSR) and OLSR. AODV and OLSR are evaluated in urban scenario where the authors enhanced HELLO and TC interval of OLSR and observe that overall enhanced OLSR performs better than AODV in urban environments.

In the paper [9] an evaluation of AODV performance in VANET is realized by varying the number of nodes maintaining the maximum velocity using 802.11 .

Our work evaluates OLSR and AODV using 802.11p and 802.11a protocols in realistic urban scenario. Moreover, five metrics performances are selected for evaluating performance of routing protocols in VANETs

\section{Routing Protocols}

The ad hoc network protocols have as main objective to reduce energy consumption and the signaling messages. Such protocols search to find the better route between a source-destiny node by the information existing in the links of the network In Vehicular Ad Hoc Networks (VANET), it is a great challenge to develop a protocol that copes with its characteristics and meets several applications requirements. With the exchange of signalling messages, it is possible to cope with the topology changes of the network. Thus, each node in the network has, previously, a routing table containing the next hop or hops forward to the destiny node.

Figure 1, based in [10], displays a brief classification of the routing protocols used in VANETs. The topology-based routing is divided in proactive and reactive mode. 


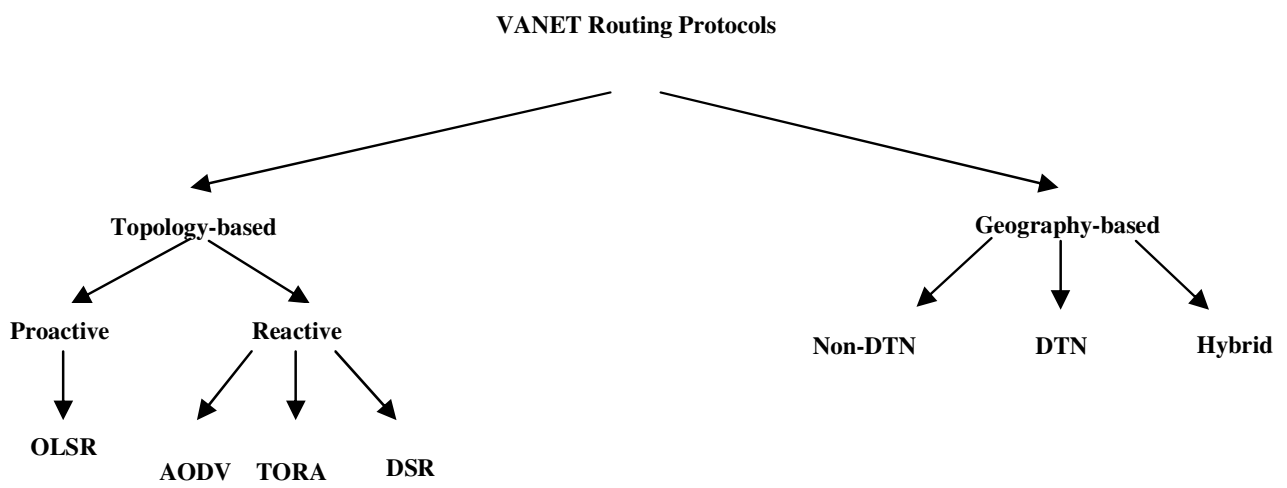

Figure 1. Brief classification of VANET routing protocol

\subsection{Proactive routing (Table-driven) protocols}

Proactive routing protocols or table-driven routing only create paths when it is necessary to forward a packet and it is not necessary to know all routes to all destiny nodes in the network. These protocols require each node to maintain one or more tables to store routing information, and they respond to change in network topology by propagating route update throughout the network to maintain consistent network view. We can cite as examples of proactive protocol: OLSR

\section{I) OLSR Protocol}

The OLSR protocol [11] is based in topology information that works in a proactive mode and it is one of the main routing protocols for ad hoc network. Each node periodically constructs and maintains the set of neighbors that can reach in 1-hop and 2-hops. Furthermore, it presents an extension called MPR (Multi Point Relay) [12,13], that minimizes the number of active relays needed to achieve 2-hops neighbors avoiding flooding, reducing the signalling messages as can be seen in the Figure 2.

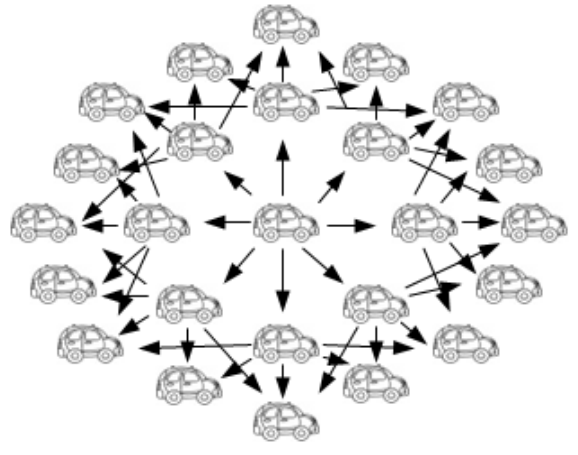

(a)

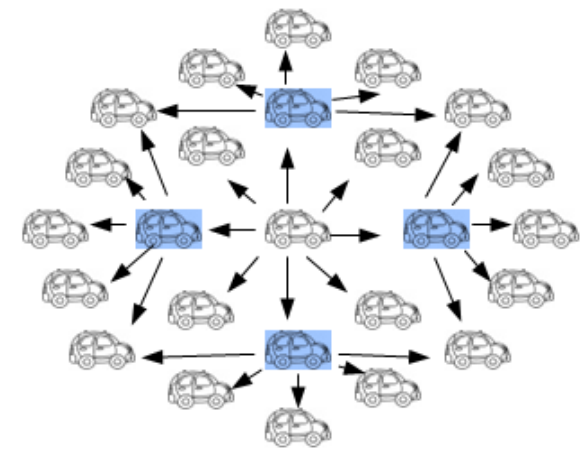

(b)

Figure 2. Types of flooding: traditional flooding (a) and flooding using MPRs (b) 


\subsection{Reactive (On-Demand) routing protocols}

In reactive (or on demand) routing protocols, the routes are created when required. When a source node wants to send to a destination node, it invokes the route discovery mechanism to find the path to the destination. This process is completed when once a source is found or all possible route permutation has been examined. Once a route has been discovered and established, it is maintained by some form of route maintenance procedure until either the destination becomes inaccessible along every path from the source or route is no longer desired.

\section{I) AODV Protocol}

AODV (Ad-hoc On-demand Distance Vector) [14] is a loop-free routing protocol for ad-hoc networks based on vector distance algorithm, as DSDV [15]. It is designed to be self-starting in an environment of mobile nodes, withstanding a variety of network behaviours such as node mobility, link failures and packet losses.

The AODV protocol is based in topology information that works in a reactive mode, in other words, when a source node wants to send data to a destination node, a route discovery process is started. In this process, the source node broadcasts a ROUTE REQUEST (RREQ) packet to all your neighbors. Neighbors nodes which do not know an active route to destination node, forward the packet to their neighbors until an available route is found or the maximum number of hops is reached. When an intermediate node knows an active route to the requested destination node, it sends back a ROUTE REPLY (RREP) packet to the source node in unicast mode, enabling to the node creates the route among the source and destination nodes. The main problem of the AODV protocol in VANET environments is the route instability due to high mobility of the nodes, breaking established paths constantly. Consequently, more packets are discarded and overhead generated due to notifications of path breaks increase significantly.

\section{Performance evaluation}

This section presents the evaluation of the AODV and OLSR protocols under 802.11a and $802.11 \mathrm{p}$ in an urban realistic scenario. The simulations were realized in the NS-2 simulator version 2.33 [16]. We consider 3 different mobility scenarios, where the number of vehicles varies from as follows: 40 vehicles (low density), 80 vehicles (medium density) and 120 vehicles (high density). Table 1 summarizes the configuration of the simulation.

All vehicles are assumed to have a radio range of 350 meters and they are moving in the urban scenario at a maximum speed of 20 meters per second. Vehicles are inserted in the map at a rate of one vehicle per second and to better characterize a realistic urban scenario, there are trafficlights in the intersections and various lanes. Vehicles are launched in the map at a rate of one new vehicle per second using random positions. When the vehicles mobility starts, the map is totally empty, and the path requests start at the hundredth second (this means that the routing protocols are only started after having a reasonable amount of running vehicles). 
International Journal of Computer Networks \& Communications (IJCNC) Vol.5, No.6, November 2013

Table 1: Mobility scenarios and parameters set considered in the simulation

\begin{tabular}{|c|c|c|c|c|}
\hline Scenario & $\begin{array}{c}\text { Number of } \\
\text { Vehicles }\end{array}$ & $\begin{array}{c}\text { Maximum } \\
\text { Speed (m/s) }\end{array}$ & $\begin{array}{c}\text { Radio range } \\
\text { (meters) }\end{array}$ & $\begin{array}{c}\text { Simulation } \\
\text { time } \\
\text { (seconds) }\end{array}$ \\
\hline Scenario 1 & 40 & 20 & 350 & 437 \\
\hline Scenario 2 & 80 & 20 & 350 & 424 \\
\hline Scenario 3 & 120 & 20 & 350 & 561 \\
\hline \multicolumn{3}{|c|}{ Two Ray Ground } \\
\hline \multicolumn{2}{|c|}{ Propagation model } & \multicolumn{3}{c|}{ 2 Mbps } \\
\hline \multicolumn{2}{|c|}{ Unicast traffic } & \multicolumn{3}{c|}{}
\end{tabular}

The scenario of simulated urban VANET was generated using TraNS tool [17] that integrates SUMO tool [18] and it was extracted from the TIGER/Line database of the US Census Bureau [19]. Such scenario has a dimension of $4100 \mathrm{~m} \times 3100 \mathrm{~m}$ area as illustrated in the Figure 3.

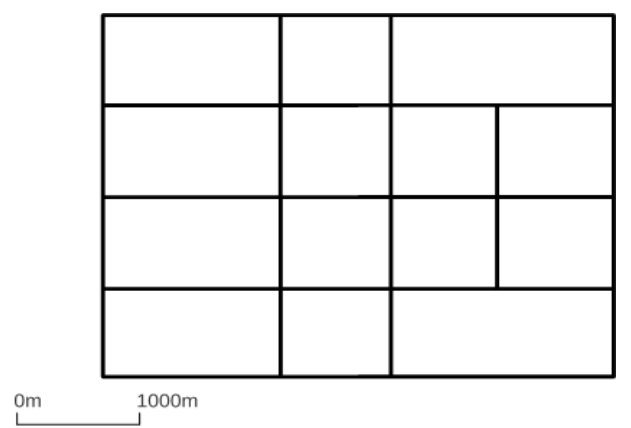

Figure3. Map of a Manhattan region, used in the realistic simulation urban scenario

Considering that all vehicles present in the map are able to route packets according to the routing protocol being evaluated, the generation of the packets is divided in two phases:

- $\quad 1^{\text {st }}$ phase: a packet is randomly destined to one of the active mobile nodes. This is the packet responsible for the path creation if the routing protocol is reactive;

- $\quad 2^{\text {nd }}$ phase: after the path being created, the source node periodically generates packets to the same destination marked in the first phase (the period was set to 1s). The node stops the packet generation when the original path breaks.

The results presented in this paper are averaged of 10 simulations for each mobility scenario and for each protocol and each communication pattern. All simulations results presented were obtained guaranteeing a $95 \%$ confidence interval.

\subsection{Performance Metrics}

As showed in the Table 1, the performance of the routing protocols AODV and OLSR with $802.11 \mathrm{p}$ and 802.11a MAC protocols was realized by varying the network density. The metrics to assess the performance are given as follows:

1. Path Availability: It is an available path between source node and destination node. 
2. $\quad$ End-to-End Delay: To the path $P=\left\{n_{1}, n_{2}, \ldots, n_{\mathrm{k}}\right\}$ where $n_{l}$ is the source node and $n_{k}$ is the destination node. Assuming that the packet is sent from node $n_{l}$ at the moment $t^{\prime}{ }_{1}$ and it is received by node $n_{k}$ at the moment $t^{\prime}{ }_{k}$, the delay is marked as $t^{\prime}{ }_{1}-t^{\prime}{ }_{k}$.

3. Number of Created Paths: It is sum of all created paths during the simulation.

4. Path Length: A path $P=\left\{n_{1}, n_{2}, \ldots, n_{\mathrm{k}}\right\}$ of $k$ nodes at the instant $t_{l}$, the path length is set by the number of hops that a packet realizes between a node source $n_{l}$ and a destination node $n_{k}$. The final value about this metric is given by $\mathrm{k}-1$ hops.

5. Path Duration: Considering again a path $P=\left\{n_{1}, n_{2}, \ldots, n_{\mathrm{k}}\right\}$ of $k$ nodes at the instant $t_{1}$, the path duration is set as the length of the major interval of time $\left[t_{1}, t_{2}\right]$, while $P$ sequence is maintained.

\section{RESULTS AND ANALYSIS}

To visualize the results, they are showed in the Figures as follows and such results are discussed for each performance metric evaluated.

- $\quad$ Path availability: The results showed in the Figure 4 demonstrate that AODV and OLSR protocols have better performance when $802.11 \mathrm{p}$ is used in all scenarios evaluated when compared with 802.11a. By using 802.11p, AODV and OLSR protocols have practically the same results.

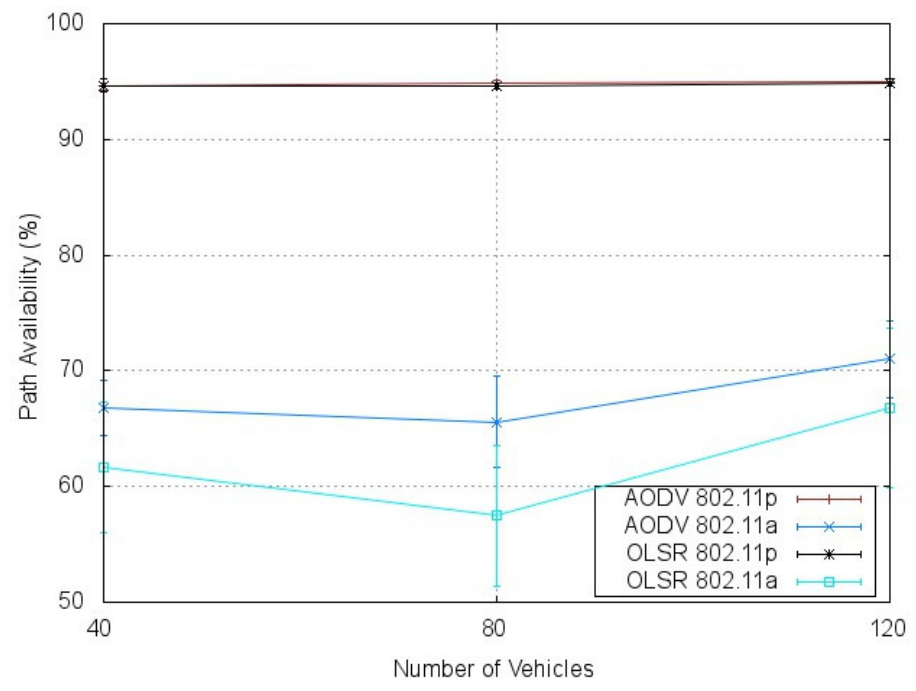

Figure 4. Path availability of the AODV and OLSR protocols using 802.11a and 802.11p

- $\quad$ End-to-End Delay: In the Figure 5, as can be seen, OLSR protocol when use $802.11 \mathrm{p}$ outperforms 802.11a. Considering that delay is a crucial factor to be considering in VANETs, the results show that using $802.11 \mathrm{p}$ is more efficient than 802.11a. AODV has poor performance as the density increases where the cost of repeated route discovery procedures in AODV introduces a large control traffic overhead and OLSR using 802.11p ends up outperforming AODV in almost $150 \%$ when AODV is using 802.11p and about $300 \%$ when AODV is using 802.11a. 
International Journal of Computer Networks \& Communications (IJCNC) Vol.5, No.6, November 2013

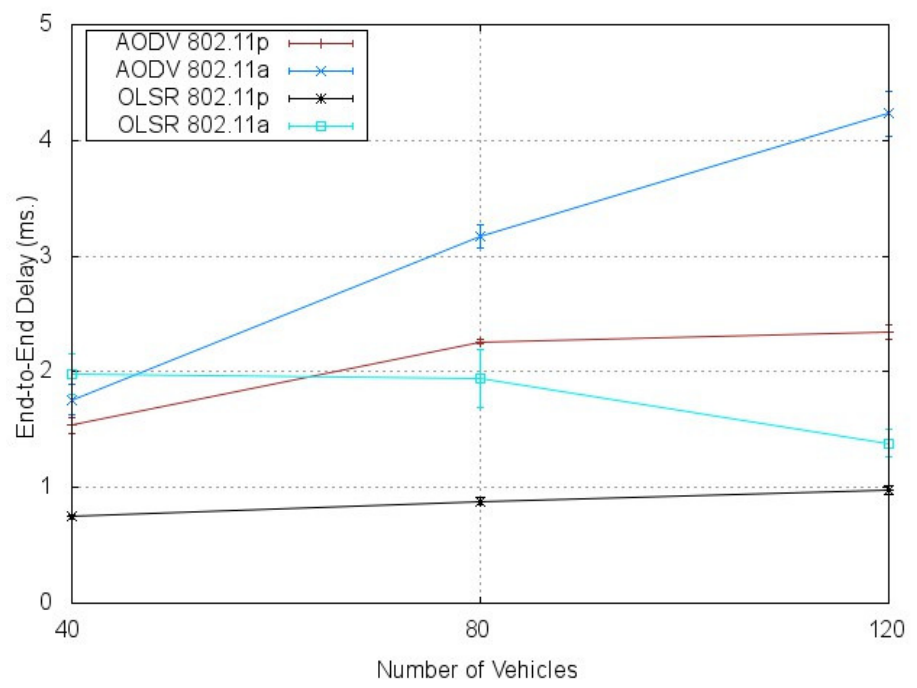

Figure 5. End-to-End Delay of the AODV and OLSR protocols using 802.11a and 802.11p

- $\quad$ Number of Created Paths: In the Figure 6 is presented the results of the number of created paths by the OLSR and AODV protocols using 802.11p and 802.11a. The results show that using AODV and OLSR protocols using 802.11p, more paths are created and consequently, more cars can communicate with each other. AODV has better results due to the fact to use flooding technique, getting more information about the network and creating more paths. Such technique has more bandwidth consumption and collisions, consequently, increasing delay.

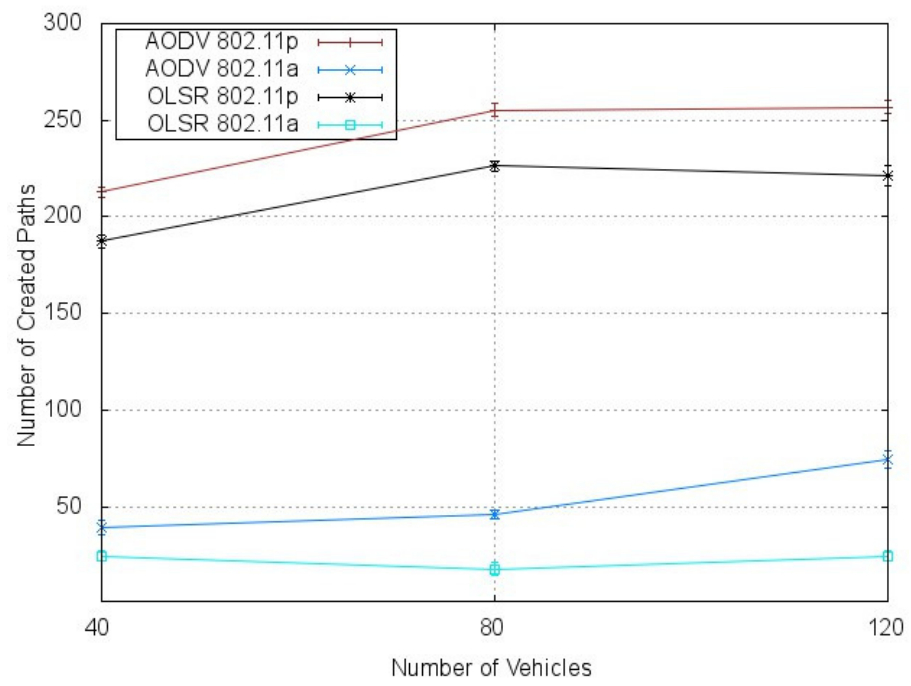

Figure 6. Number of Created Paths of the AODV and OLSR protocols using 802.11 and $802.11 \mathrm{p}$

- $\quad$ Path Length: AODV protocol has poor results using 802.11a and 802.11p. More number of hops leads to higher probability of broken links. Then, OLSR protocol has more stables paths, with minor probability of broken links. 
International Journal of Computer Networks \& Communications (IJCNC) Vol.5, No.6, November 2013

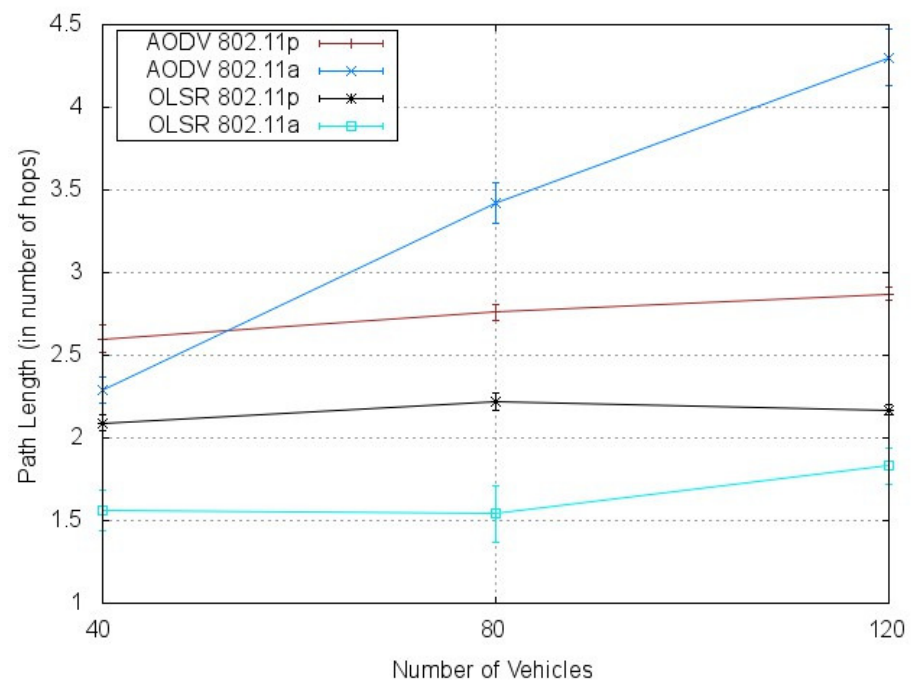

Figure 7. Path Length of the AODV and OLSR protocols using 802.11a and 802.11p

- $\quad$ Path Duration: Finally, Figure 8 shows the path duration. OLSR protocol uses MPR nodes, reducing signaling messages and getting better results when compared with AODV, mainly in scenario with medium vehicle density.

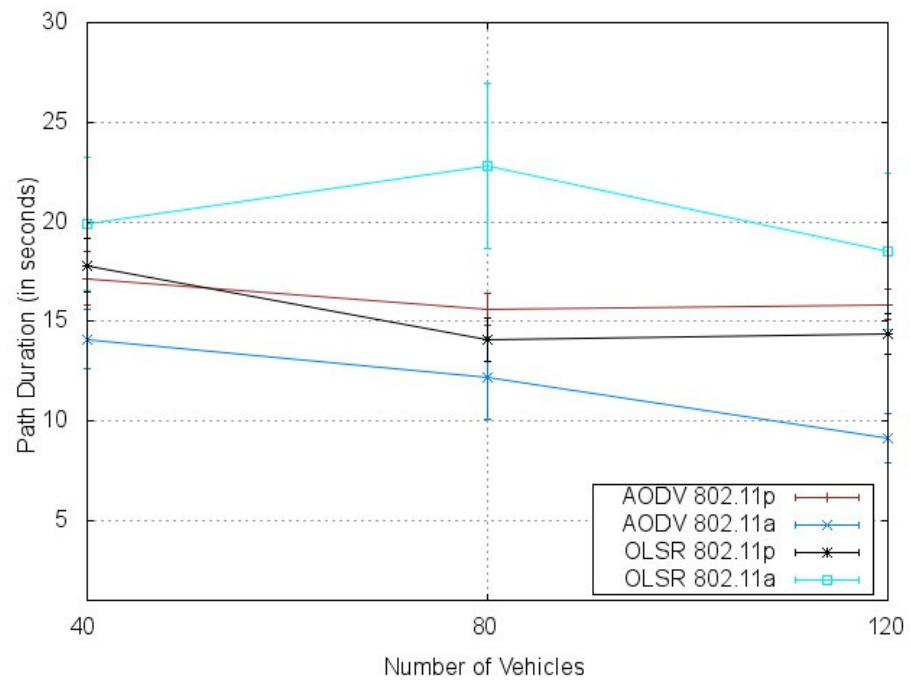

Figure 8. Path Duration of the AODV and OLSR protocols using 802.11a and 802.11p

\section{Conclusions}

In this paper, we evaluate AODV and OLSR topology-based protocols under 802.11a and $802.11 \mathrm{p}$ in an urban scenario considering different vehicle density. VANETs have a high dynamic topology, where AODV protocol suffers to maintain routing table constantly updated increasing signalling control messages. For most of the metrics evaluated in this paper, OLSR has better performance that AODV, mainly when $802.11 \mathrm{p}$ is used showing that OLSR can be an alternative to use in VANETs. 
As future works, the authors are also interested in evaluating routing protocols for VANET in urban scenarios with other realistic characteristics such as accidents, overtaking, points of interest and higher number of nodes.

\section{ACKNOWLEDGEMENTS}

The authors would like to thank professor Larissa Broetto by revision the article and giving some suggestions.

\section{REFERENCES}

[1] The FCC DSRC web site. http://wireless.fcc.gov/services/its/dsrc/

[2] Bryan Parno, Adrian Perrig, "Challenges in Securing Vehicular Networks", Poster presented at USENIX Security Symposium,August-04.

[3] Szczurek P, Xu B, Wolfson O, Lin J. A methodology for the development of novel, vanet safety applications. Proceedings of the ninth ACM international workshop on Vehicular internetworking, systems, and applications, June 2012; 119-122.

[4] Vaghela VB, Shad DJ. Vehicular parking space discovery with agent approach. Proceedings of the International Conference and Workshop on Emerging Trends in Technology. February 2011; 613617

[5] Huang CM, Lin SY. An early collision warning algorithm for vehicles based on v2v communication. International Journal of Communications Systems 2012; 25(6): 779-795.

[6] Chennikara, J., Chen, W., Altintas, O., \& Cai, S. (2001). Survey of routing protocols for intervehicle communications. Trird Annual International Conference Mobile and Ubiquitous Systems: Networking and Services, 1-5.

[7] Manvi, SS et al., "Performance Analysis of AODV, DSR, and Swarm Intelligence Routing Protocols In Vehicular Ad hoc Network Environment", ICFCC, 2009

[8] Nadeem Javaid, Ayesha Bibi, Safdar Hussain Bouk, Akmal Javaid, and Iwao Sasase. Modeling enhancements in DSR, FSR, OLSR under mobility and scalability constraints in VANETs. ICC, page 6504-6508. 2012

[9] Khan, I. and Qayyum, A., "Performance evaluation of AODV and OLSR in highly fading vehicular ad hoc network environments", INMIC, 2009

[10] "Survey of Routing Protocols in Vehicular Ad Hoc Networks," Kevin C. Lee, Uichin Lee, Mario Gerla, Advances in Vehicular Ad-Hoc Networks: Developments and Challenges, IGI Global, Oct, 2009.

[11] Jacquet, P., Muhlerthaler, P., Clausen, T., et al (2001). Optimized link state routing protocol for ad hoc networks. IEEE International Multi Topic Conference, 62-68

[12] Saadi Boudjit. "Autoconfiguration Algorithm For a Multiple Interfaces Adhoc Network Running OLSR Routing Protocol". International Journal of Computer Networks and Communications (IJCNC). Vol. 5, No. 1, January 2013, 153-170.

[13] Emanuel Bacelli, Juan Antonio Cordero and Philippe Jacquet. "OSPF over Multi-Hop Ad Hoc Wireless Communications". International Journal of Computer Networks and Communications (IJCNC). Vol. 2, No. 5, September 2010, 37-56.

[14] Perkins, C.E., \& Royer, C.M. (1999). Ad-hoc on-demand distance vector routing. Second IEEE Workshop on Mobile Computing Systems and Applications, 90-100.

[15] Mahdipour, E. Rahmani, A.M. Aminian, E. ," Performance Evaluation of Destination-Sequenced Distance-Vector (DSDV) Routing Protocol”, Proceeding March 2009 , page(s): 186 - 190, ISBN: 978-0-7695-3567-8

[16] Information Sciences Institute (2011). NS-2 Network Simulator (version 2.33). Available at http://nsnam.isi.edu/nsnam/index.php/Main Page

[17] TraNS (2011), Open source tool for realistic simulations of VANET applications. Available at http://trans.ep.ch/

[18] Krajzewicz, D.; Hertkorn, G.; Wagner, P. et al (2002)., Sumo (simulation of urban mobility), an open-source trace simulation. 4th Middle East Symposium on Simulation and Modelling (MESM2002), 183-187. 
International Journal of Computer Networks \& Communications (IJCNC) Vol.5, No.6, November 2013

[19] U.S. Census Bureau (2011). TIGER database / Line - Second edition. Available at: http://www.census.gov/geo/www/tiger

\section{Authors}

Ederval Pablo Ferreira da Cruz received the graduation in Data Processing from the University Center of Espirito Santo (UNESC), Brazil, in 2001 and the M.Sc degree from the Federal University of State of Rio de Janeiro (UNIRIO), Brazil, in 2012. He is currently professor of the Federal Institute of Espirito Santo (IFES) - Campus Itapina. His research interests include vehicular networks and wireless sensor networks

Bruno dos Santos Silva is an undergraduate student at Federal University of State of Rio de Janeiro (UNIRIO). Currently, he has a scholarship from CNPq at National Institute for Pure and Applied Mathematics (IMPA).
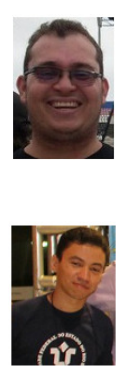